\title{
The realization of low order FSM method and its application
}

\author{
Jiai $\mathrm{He}^{1, \mathrm{a}}$, Xiangyang $\mathrm{Liu}^{1, \mathrm{~b}}$, Chengquan $\mathrm{Pei}^{2,3, \mathrm{c}}$ \\ ${ }^{1}$ School of Computer and Communication, Lanzhou Univ. of Tech, Lanzhou 730050, China; \\ ${ }^{2}$ Xi'an Jiaotong University, Shanxi, xi'an, 710000, China; \\ ${ }^{3}$ Xi'an Institute of Optics and Precision Mechanics of CAS, shanxi, xi'an, 710000, China. \\ ahejiai@lut.cn, 'Ixiangyang1206@126.com, Pcq4292669@126.com
}

Keywords: $\alpha$ stable distribution; FLOS; low order FSM algorithm

\begin{abstract}
Cyclostationary statistical characteristic is the main theory of studying non-stationary periodic signal. Signal and noise are always assumed that obey Gaussian distribution model in traditional signal's processing. As the environment of non-Gaussian pulse is increasing complex, degradation problems of system performance can be solved by building $\alpha$ stable distribution theory model. Firstly, this paper introduces the $\alpha$ stable distribution to estimate FSM cyclic spectrum and proposes cycle spectrum analysis under discrete signal. Then, the verification is given by taking AM communication signal on Matlab platform. The results show that in a complex environment, the low-level FSM algorithm which is based on $\alpha$ stable distribution has better performances on robustness and noise immunity. Finally, the algorithm proposed in this paper is applied to construct characteristic parameter, which is important for blind signal separation and identification.
\end{abstract}

\section{I .Introduction}

As the Gaussian assumption based on the central limit theorem conform to the normal situation, and its description is simple, analysis and processing are also convenient, many principles and methods of traditional research areas are often assumed to be Gaussian model, such as signal characteristic analysis, signal filtering, parameter estimation and detection, system identification etc., and Gaussian distribution model is always popular in the field. But in practical applications, the signals we meet are often been accompanied by some noises which have low probability and loud magnitude. If we use a Gaussian distribution model to describe such processes, the signal processor's performance will be significantly degraded for the noise cannot match with the model well. Therefore, we have to consider using non-Gaussian signal noise model and design a processing system, so as to make them in line with signal's noise characteristics better.

$\alpha$ stable distribution is a kind of generalized Gaussian distribution ${ }^{[1-3]}$, and it is also a distribution that can satisfy generalized central limit theorem. It becomes the most potential and attractive model to describe random signals which have more significant spike pulse waveform and thicker tail probability density function for its better robustness performance. $\alpha$ stable distribution can describe various degrees of impulse noise, which is based on setting different characteristic parameters, no matter the noise is symmetrical or asymmetrical. This article promotes the FSM algorithm to $\alpha$ stable distribution, makes the simulation confirmation to FSM algorithm under the stable distribution, and presents the system block diagram. It provides a new way for signal separation, recognition and extraction under impulse noise environment.

\section{II .Fractional lower order cyclic spectral density}

The statistical moments of signal contain a wealth of feature information .Fractional lower order statistics theory(FLOS) [4,5]is a powerful tool for studying $\alpha$ stable distribution. Hypothesis $x(t), t \in(-\infty, \infty)$ is a real SaS distributed random process whose characteristics index is $\alpha$,and position parameter is 0.Its traditional second-order autocorrelation function is: 


$$
R(t, \tau)=E[x(t) x(t+\tau)]
$$

If $x(t)$ is a cyclostationary signal that meet the following relation ( $T$ is a cycle )

$$
R(t, \tau)=R(t+T, \tau)
$$

The second-order statistical moments of the signal is not exist when it is mixed with stable distribution impulse noise. Therefore, fractional lower order cycle stationary signal can be defined as (3):

$$
R_{x x}(t, a ; t+\tau, b)=E[x(t, a) x(t+\tau, b)]=R_{x x}(t+T, a ; t+T+\tau, b)
$$

In addition: $x(t, a)=[x(t)]^{\langle a\rangle}, 0<a<\alpha / 2$. According to Fourier series expansion:

$$
R_{x x}^{\varepsilon}(\tau, a, b)=\frac{1}{T} \int_{-T / 2}^{T / 2} R_{x x}(t, a ; t+\tau, b) e^{-j 2 \pi \varepsilon t} d t
$$

Among them $\varepsilon$ is a cyclic frequency. As the power spectrum and autocorrelation function are Fourier transform pairs, we define the fractional lower order cyclic spectral density as following :

$$
S_{x x}^{\varepsilon}(f ; a, b)=\int_{-\infty}^{\infty} R_{x x}^{\varepsilon}(\tau ; a, b) e^{-j 2 \pi f \tau} d \tau
$$

The modulated signal is cyclostationaritic. Through analysis the spectral correlation structure of the signal, we can complete a variety of signal processing tasks, such as blind signal detection, modulation recognition and classification, parameter estimation and blind equalization etc. .

\section{Low-level FSM algorithm}

Assume ${ }^{x(t)}$ is a second-order cyclostationary process, the cyclic spectrum based on continuous FSM algorithm has the following expression[6,7]:

$$
\begin{aligned}
& X_{T}(t, f)=\int_{t-T / 2}^{t+T / 2} x(u) e^{-j 2 \pi f u} d u \\
& S_{x_{T}}^{\alpha}(t, f)=\frac{1}{T} X_{T}\left(t, f-\frac{\alpha}{2}\right) X_{T}^{*}\left(t, f+\frac{\alpha}{2}\right)
\end{aligned}
$$

We obtain the corresponding discrete FSM algorithm as following ${ }^{[8]}$ :

$$
S_{X_{\Delta t}}^{\alpha}(t, f)_{\Delta f}=\frac{1}{M} \sum_{v=-(M-1) / 2}^{(M-1) / 2} \frac{1}{\Delta t} X_{\Delta t}\left(t, f+\frac{\alpha}{2}+v F_{s}\right) \cdot X_{\Delta t}^{*}\left(t, f-\frac{\alpha}{2}+v F_{s}\right)
$$

Where $X_{\Delta t}(t, f)$ has the following form:

$$
X_{\Delta t}(t, f)=\sum_{k=0}^{N-1} a_{\Delta t}\left(k T_{s}\right) x\left(t-k T_{s}\right) e^{-j 2 \pi f\left(t-k T_{s}\right)}
$$

Among them $a_{\Delta t}\left(k T_{s}\right)$ is a window for the attenuation data, $\Delta f=\mathrm{MF}_{\mathrm{s}}$ is the width of the spectrum smoothing, $\mathrm{F}_{\mathrm{s}}=1 / \mathrm{NT}_{\mathrm{s}}$ is the frequency domain sampling width, $\mathrm{T}_{\mathrm{s}}$ is the time domain sampling width, and $\mathrm{N}_{\text {is }}$ the total number of samples in the $\Delta \mathrm{t}, \mathrm{N}=\Delta \mathrm{t} / \mathrm{T}_{\mathrm{s}}+1$. The second-order statistical moments of the signal is not exist when it is mixed with stable distribution impulse noise. Therefore, the low-level statistical features under stable distribution is introduced in (4) 、 (5), the result as following:

Discrete case $^{[9]}$ :

$$
\begin{aligned}
X_{T}^{(A)}(t, f) & =\int_{t-T / 2}^{t+T / 2} x^{\langle A\rangle}(u) e^{-j 2 \pi f u} d u, \quad A=\frac{p}{2} \\
S_{X_{T}, p}^{\alpha}(t, f) & =\frac{1}{T} X_{T}^{\langle A\rangle}\left(t, f+\frac{\alpha}{2}\right) X_{T}^{*\langle A\rangle}\left(t, f-\frac{\alpha}{2}\right)
\end{aligned}
$$




$$
S_{X_{\Delta t, p}}^{\alpha}(t, f)_{\Delta f}=\frac{1}{M} \sum_{v=-(M-1) / 2}^{(M-1) / 2} \frac{1}{\Delta t} X_{\Delta t}^{(A)}\left(t, f+\frac{\alpha}{2}+v F_{s}\right) \cdot X_{\Delta t}^{*(A)}\left(t, f-\frac{\alpha}{2}+v F_{s}\right)
$$

Among them $\mathrm{X}_{\Delta \mathrm{t}}{ }^{(\mathrm{A})}(t, f)$ has following form:

$$
X_{\Delta t}^{(A)}(t, f)=\sum_{k=0}^{N-1} a_{\Delta t}\left(k T_{s}\right) x^{\langle A\rangle}\left(t-k T_{s}\right) e^{-j 2 \pi f\left(t-k T_{s}\right)}
$$

All of the parameters are consistent with the Gaussian model.

\section{Examples analysis}

We take modulation signal $f(t)=a(t) \cos \left(2 \pi f_{c} t\right)$ for example to analyze low-level circulation spectrum:

$$
F_{\Delta t}^{(A)}(t, v)=\int_{t-\Delta t / 2}^{\mathrm{t}+\Delta t / 2}\left[a(t) \cos \left(2 \pi f_{c} t\right)\right]^{\langle A\rangle} e^{-j 2 \pi f u} d u
$$

If $^{a(t)}$ is a complex signal,

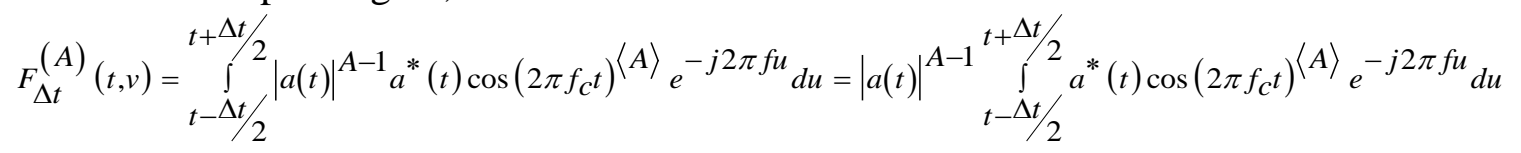

Therefore: $S_{f \Delta t, p}^{\alpha}(f)=\lim _{\Delta f \rightarrow 0} \lim _{\Delta t \rightarrow \infty} \frac{1}{\Delta f} \int_{f_{-} \Delta f / 2}^{f+\Delta f / 2} \frac{1}{\Delta t}|a(t)|^{p} F_{\Delta t}^{(A)}\left(t, f-\frac{\alpha}{2}\right) \cdot F_{\Delta t}^{*(A)}\left(t, f+\frac{\alpha}{2}\right) d f$

$$
\text { If } a(t) \text { is a real signal, }
$$

$$
F_{\Delta t}^{(A)}(t, v)=\int_{t-\Delta t / 2}^{t+\Delta t / 2}[f(t)]^{\langle A\rangle} \cos \left(2 \pi f_{c} t\right)^{\langle A\rangle} e^{-j 2 \pi f u} d u=\int_{t-\Delta t / 2}^{t+\Delta t / 2}|f(t)|^{A} \operatorname{sgn}(t) \cos \left(2 \pi f_{c} t\right)^{\langle A\rangle} e^{-j 2 \pi f u} d u
$$

As $0<p \leq 2, \mathrm{~A}=\mathrm{P} / 2,0<A \leq 1$, Make the following change:

$$
f(t) * g(t)=\hat{f(t)} \quad g(t)=\frac{1}{\pi t}
$$

Assume: $f^{\prime}(t)=f(t)+j \hat{f(t)}$, Therefore $f(t)=\operatorname{Re}\left[f^{\prime}(t)\right]$ form.

We can obtain the same spectral function with the plural form by turning the real form into plural In the discrete case, $f(t)=a(t) \cos \left(2 \pi f_{c} t\right)$, Assume $a(t)$ is a complex signal, $T_{s}$ is the sampling interval,

$$
F_{\Delta t}^{(A)}\left(t, f_{s}\right)=\sum_{k=0}^{N-1} a_{\Delta t}\left(k T_{s}\right) f^{\langle A\rangle}\left(t-k T_{s}\right) e^{-j 2 \pi f\left(t-k T_{s}\right)}
$$

Therefore:

$$
\begin{aligned}
S_{x \Delta t, p}^{\alpha}(t, f)_{\Delta f}= & \frac{1}{M} \sum_{v=-(M-1) / 2}^{(M-1) / 2}\left[\frac{1}{\Delta t} \sum_{k=0}^{N-1} a_{\Delta t}\left(k T_{s}\right) \cdot\left|f\left(t-k T_{s}\right)\right|^{A-1} f^{*}\left(t-k T_{S}\right) e^{-j 2 \pi\left(f-\frac{\alpha}{2}\right)\left(t-k T_{s}\right)}\right] . \\
& {\left[\sum_{k=0}^{N-1} a_{\Delta t}\left(k T_{s}\right)\left|f\left(t-k T_{s}\right)\right|^{A-1} f^{*}\left(t-k T_{S}\right) e^{-j 2 \pi\left(f+\frac{\alpha}{2}\right)\left(t-k T_{s}\right)}\right]^{*} }
\end{aligned}
$$

The system block diagram is shown in figure 1: 


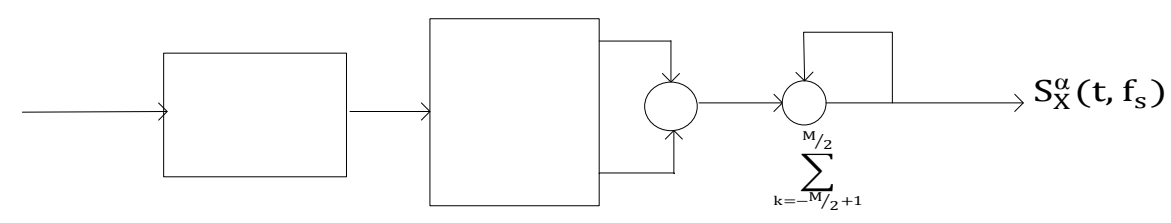

Figure 1 A low-level FSM algorithm system realization diagram

\section{V . Feature extraction based on low-level cycle spectrum}

It is not difficult to get other modulated signals' cyclic spectrum expression under stable distribution through the above mathematical analysis. we can select the following characteristic parameters based on low-level cyclic spectrum :

1 ) the amplitude of cycle spectrum $S_{x}^{a=2 f_{c}+1 / T_{s}}(f=0)$ :

$$
\begin{array}{ll}
\left|S_{B P S K}^{a=2 f_{c}+1 / T_{s}}(f=0)\right|=k_{p}\left|Q\left(1 / 2 T_{s}\right) Q^{*}\left(-1 / 2 T_{s}\right)\right| / 4 T_{S} & \left|S_{B P S K}^{a=2 f_{c}+1 / T_{s}}(f=0)\right|=0 \\
\left|S_{\text {OQPSK/MSK }}^{a=2 f_{c}+1 / T_{S}}(f=0)\right|=k_{p}\left|Q\left(1 / 2 T_{s}\right) Q^{*}\left(-1 / 2 T_{s}\right)\right| / 2 T_{S} &
\end{array}
$$

2) the amplitude of cycle spectrum $S_{x}^{a=1 / T_{s}}\left(f=f_{c}\right)$ :

$$
\left|S_{\text {BPSK IMPSK }}^{a=1 / I_{s}}\left(f=f_{c}\right)\right|=k_{p}\left|Q\left(1 / 2 T_{s}\right) Q^{*}\left(-1 / 2 T_{s}\right)\right| / 4 T_{S} \quad\left|S_{\text {OQPSK } / M S K}^{a=1 / T_{S}}\left(f=f_{c}\right)\right|=0
$$

3 ) the amplitude of cycle spectrum $S_{x}^{a=2 / T_{s}}\left(f=f_{c}\right)$ :

$$
\left|S_{B P S K / M P S K}^{a=2 / T_{s}}\left(f=f_{c}\right)\right|=k_{p}\left|Q\left(1 / T_{s}\right) Q^{*}\left(-1 / T_{s}\right)\right| / 4 T_{S} \quad \quad\left|S_{\text {OQPSK } / M S K}^{a=2 / T_{s}}\left(f=f_{c}\right)\right|=k_{p}\left|Q\left(1 / T_{s}\right) Q^{*}\left(-1 / T_{s}\right)\right| / 2 T_{S}
$$

From the above theoretical analysis, we know that $k_{p}$ is a constant related to $\mathrm{p}$, and build up following characteristic parameters based on low-level cyclic spectral:

\begin{tabular}{|c|c|c|c|}
\hline & BPSK & QPSK/8PSK & MSK/OQPSK \\
\hline \multirow[b]{2}{*}{$A_{1}$} & $\left|Q\left(1 / 2 T_{S}\right) Q^{*}\left(-1 / 2 T_{S}\right)\right|$ & $Q\left(1 / 2 T_{S}\right) Q^{*}\left(-1 / 2 T_{S}\right) \mid$ & \multirow[b]{2}{*}{0} \\
\hline & $\left|Q\left(1 / T_{s}\right) Q^{*}\left(-1 / T_{s}\right)\right|$ & $\left|Q\left(1 / T_{S}\right) Q^{*}\left(-1 / T_{S}\right)\right|$ & \\
\hline
\end{tabular}

$$
A_{1}=\left|\frac{S_{x}^{a=\frac{1}{T_{s}}}\left(f=f_{c}\right)}{S_{x}^{a=\frac{2}{T_{s}}}\left(f=f_{c}\right)}\right| \quad A_{2}=\left|\frac{S_{x}^{a=\frac{1}{T_{s}}}\left(f=f_{c}\right)}{S_{x}^{a=2 f_{c}+\frac{2}{T_{s}}}(f=0)}\right|
$$

Through analysis, signal's characteristic parameters as shown in table 1:

Table1 the characteristic parameters of different modulation signals

$\mathrm{A}_{2}$ 1 $\infty \quad 0$

The result showing in the table 1, the characteristic parameter A1 of BPSK and MPSK is same, and is always more than 1 , while the characteristic parameter A1 of MSK and OQPSK is 0 . We can separate the BPSK/MPSK and MSK/OQPSK by setting the threshold th $1=1$. Similarly, for the characteristic parameters A2, the BPSK and QPSK/8PSK also can be separated by setting the threshold th2 $=2$. We can identify different modulated signals by designing the above classification, which structure is simple, but has a good performance in complex environment. Therefore, the low order cyclic spectrum analysis of the modulation signals in this paper offers a new approach to modulation recognition under $\alpha$ stable distribution.

\section{Computer simulation}

We make simulation for AM modulation signal, the carrier frequency $f_{c}=200 \mathrm{kHz}$, the sampling frequency is $600 \mathrm{KHz}$, the symbol rate is 256 , and the baseband signal is a single sinusoidal function. We choose conventional second-order correlation analysis method when mixing impulse noise and Gaussian noise (SNR $=5 \mathrm{~dB}$ ). The simulation results as figure 2 and figure3.Making simulation for AM modulation signal by using the algorithm proposed in this paper, the simulation results as figure 
4 and figure 5(p=1). Under Gaussian assumption, the cyclostationary of AM signal based on second-order cyclic spectral theory as figure 6.

According to theoretical analysis we can obtain that the maximum correlation peaks appear at $\alpha=0$,

$\alpha= \pm 2 f_{0}$. Figure 5(a) and Figure5(b) show that two peaks appear at the $\alpha=0$, and the other peak appears at $\alpha= \pm 130 \mathrm{KHz}$.Figure3(a), Figure3(b) show the cycle spectrum based on the traditional method affects spectral structure under steady noise distribution. And we compare Figure 3 to Figure5, the algorithm based on the assumption of $\alpha$ stable distribution has a better anti-noise performance than Gaussian assumption. The results show that the algorithm based on the assumption of stable distribution is effectiveness and reliability.
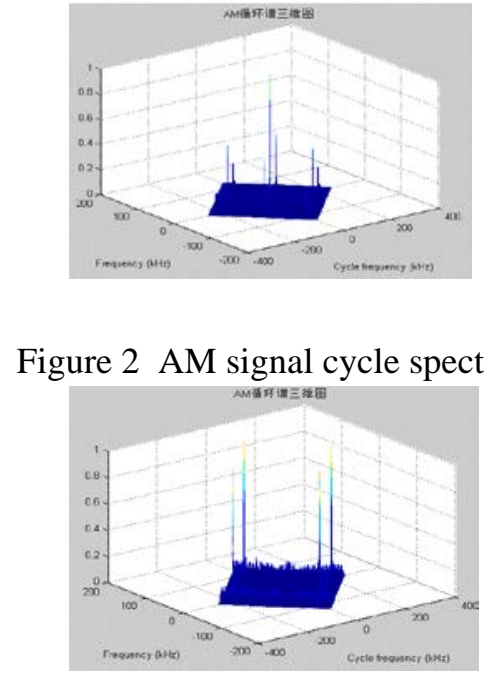

Figure 4 AM signal 3D cycle spectrum

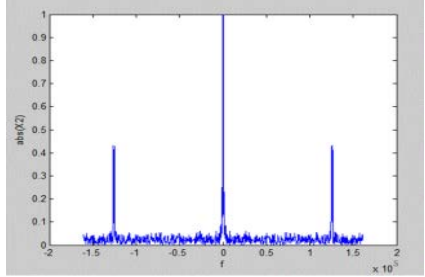

(a) $\xi=0$

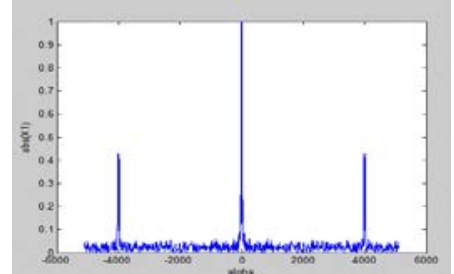

(b) $f=0$

Figure 2 AM signal cycle spectrum with mixed noise Figure 3 Sectional view of the cyclic spectrum

\section{VII .Summary}

According to the analysis above, it is clear that the structure of cyclic spectrum on the Alpha stable distribution is the same as the structure on the assumption of the Gaussian. But the magnitude is different, which mainly depend on the change of $p$. If $p=2$, the fractional cyclic spectrum is transformed into the second-order cyclic spectrum. In addition, the analysis of cyclostationarity feature based on the assumption of non-Gaussian has good anti-noise ability.The cyclic frequency or spectral structure can be regarded as a set of parameters to identify different modulation signal, which is based on the conclusion that different communication signals have different cyclic structure. Besides, the theory can be applied to the blind source separation, which is of the character of pulse noise. The cyclic frequency or new variable based on cyclostationarity can be seen as characteristic parameters to separate. In conclusion, it is very meaningful for enriching the theory of signal processing to study the character of cyclostationarity signals under stable distribution.

\section{Acknowledgement}

This work was supported by the National Natural Science Foundation of China

（No.61561031）, and the Natural Science Foundation of Gansu Province（No.148RJZA018）. For more information on low order FSM method, send the email to lxiangyang1206@126.com.

\section{References}

[1] Gardner W A. Cyclostationarity in Communications and Signal processing[R]. New York. IEEE press,1994,pp:329-454. 
[2] Chen $\mathrm{H} \mathrm{L}$, Wang $\mathrm{J} \mathrm{T}$, Zhang $\mathrm{Y}$, et al. Research on parameterizations of stable distribution[C]//Electronic Engineering and Information Science: Proceedings of the International Conference of Electronic Engineering and Information Science 2015 (ICEEIS 2015), January 17-18, 2015, Harbin, China. CRC Press, 2015: 405.

[3] Nikias C L, Shao M. Signal processing with alpha-stable distributions and applications[M]. Wiley-Interscience, 1995.

[4] Liang Y, Chen W. A survey on computing Lévy stable distributions and a new MATLAB toolbox[J]. Signal Processing, 2013, 93(1),pp: 242-251.

[5] Cek M E. Covert communication using skewed $\alpha$-stable distributions[J]. Electronics Letters, 2014, 51(1),pp: 116-118.

[6] Gardner W A. Measurement of spectral correlation [J]. IEEE Trans.Acoust Speech, Signal Processing. 1986, 34(5),pp:1111-1123.

[7] Achim A M,Canagarajah C N,Bull D R. Complex wavelet domain image fusion based on fractional lower order moments[C].IEEE International Conference on Information Fusion 2005, pp:515-521.

[8] William A.Gardner. Measurement of Spectral Correlation [J].IEEE Transcation on Acoustics, Speech and Signal Processing, Oct 1986, Vo1.34, No.5, pp:1111-1123.

[9] Gardner W A. The spectral correlation theory of cyclostationary time-series [J]. Signal Processing, 1986,11(1), pp:13-36. 